\title{
DEFICIENCIA DE VITAMINA B12 RELACIONADA AL USO DE METFORMINA ARTÍCULO DE REVISIÓN.
}

\author{
VITAMIN B12 DEFICIENCY RELATED TO METFORMIN USE \\ A REVIEW ARTICLE.
}

\author{
Arauz Valdes, Edwin*; Cardoze, Denise*; Salehji Bhana, Abdullah*; Liguas, Aillyn* \\ * Médico General, Universidad de Panamá, Ciudad de Panamá
}

Recibido: 13 de julio del 2020

Aceptado: 9 de septiembre del 2020

Arauz Valdes E, Cardoze D, Salehji Bhana A, Liguas A. Deficiencia de Vitamina B12 Relacionado al uso de Metformina Artículo de Revisión. Rev méd cient. 2020; Volumen 33 (1): página 52-63. DOI: 10.37416/rmc.v33i1.585

\section{RESUMEN}

ANTECEDENTES: La diabetes mellitus es una enfermedad metabólica de etiología diversa y alta prevalencia a nivel mundial, en el 2019 se estimó 463 millones de casos y su tendencia va en aumento. La metformina es el medicamento más utilizado en pacientes con diabetes mellitus tipo 2. La deficiencia de vitamina B12 es un efecto adverso de este, pero muy poco estudiado. Factores como: edad, sexo, dosis, duración del tratamiento, entre otros; han sido relacionados al desarrollo de esta complicación.

OBJETIVO: Recopilar y sintetizar información actualizada acerca de la deficiencia de vitamina B12 secundaria al uso de metformina, su mecanismo y los factores de riesgorelacionados.

DISEÑO: Se recolectaron artículos de revisión, trabajos de investigación y metaanálisis de bases de datos científicas como PubMed, Scielo, Cochrane y Science Direct. Los artículos seleccionados fueron publicados entre 2010 a 2020.

CONCLUSIÓN: Las recomendaciones sobre estudios de tamizaje para detectar la deficiencia de vitamina B12 a usuarios de metformina aún no son claras y no se encuentran en guías. Factores como el uso prolongado y la dosis del tratamiento están relacionados a su desarrollo. Esta población se beneficia de la monitorización y de la suplementación dependiendo del riesgo.

PALABRAS CLAVE: Metformina, Deficiencia de Vitamina B12, Vitamina B12, Absorción.

\section{ABSTRACT}

BACKGROUND: Diabetes mellitus is a metabolic disease of diverse etiology and high prevalence worldwide. In 2019, 463 million cases were estimated, a number that tends to increase by year. Metformin is the most widely used medication in type 2 diabetes mellitus. Vitamin B12 deficiency is a poorly studied adverse effect of the latter. Factors such as age, sex, dose, duration of treatment, among others have been related to the development of this complication.

OBJECTIVE: Collect and synthesize current information about vitamin B12 deficiency related to metformin use, its mechanism and related risk factors.

DESIGN: Review articles, research papers and meta-analyzes from scientific databases such as PubMed, Scielo, Cochrane and Science Direct were collected. The selected articles were published between 2010 to 2020.

CONCLUSION: Screening recommendations for vitamin B12 deficiency in metformin users are not yet clear and do not appear in guidelines. Variables such as long-term usage of metformin and its dosing are related to its development. This patient population benefits from monitoring and even supplementation, depending on the risk.

KEY WORDS: Metformin, Vitamin B12 Deficiency, Vitamin B12, Absorption.

Deficiencia de Vitamina B12 Relacionada al Uso de Metformina Artículo de Revisión by Autor is licensed under a Creative Commons Attribution-NonCommercial-NoDerivs 4.0 Unported License.

Permissions beyond the scope of this license may be available at www.revistamedicocientifica.org. 


\section{INTRODUCCIÓN}

La Diabetes Mellitus (DM) es una enfermedad metabólica de etiología diversa que se caracteriza por déficit en la secreción de insulina, resultando en hiperglicemia, que da lugar a manifestaciones clínicas y complicaciones bien descritas. ${ }^{1,2}$

Representa un problema de salud pública mundial por su alta prevalencia. En 2019 se estimó que 463 millones de adultos entre 20 y 79 años sufren de esta enfermedad y se cree que para el 2045 esta cifra aumentará a más de 700 millones de pacientes. A nivel mundial, los costos relacionados con el manejo de la DM superan los 760 mil millones de dólares. ${ }^{3}$

El tratamiento farmacológico de la DM depende de las características del paciente y de la propia enfermedad. Sin embargo, en términos generales, la metformina es considerada el agente farmacológico de primera elección en pacientes con diabetes mellitus tipo 2 (DM) 2). ${ }^{4}$

La metformina forma parte de las biguanidas y ejerce su efecto hipoglicemiante a través de la disminución de la gluconeogénesis hepática. A pesar de ser uno de los medicamentos más utilizados a nivel mundial, aproximadamente 120 millones de personas, aún existen ciertas dudas con respecto a su mecanismo de acción exacto. ${ }^{5,6}$

Los efectos adversos más frecuentemente asociados al uso de metformina se relacionan con molestias gastrointestinales como diarrea, náuseas y dolor abdominal; acidosis láctica y deficiencia de vitamina B12 ${ }^{5}$.

La vitamina B12 es un nutriente esencial que se obtiene a través de la dieta. Su proceso de absorción es complejo y consta de múltiples pasos; cualquier alteración en alguno de estos puede traducirse en el déficit de la misma. La deficiencia de vitamina B12 debe sospecharse en pacientes con manifestaciones hematológicas y neurológicas típicas, sobre todo en poblaciones de riesgo. ${ }^{7}$

Existen distintas teorías que tratan de explicar el mecanismo mediante el cual la metformina produce déficit de vitamina B12. La de mayor aceptación se basa en la interferencia de esta biguanida en su absorción dependiente de calcio a nivel del íleo terminal. ${ }^{8}$

Considerando el impacto global de la DM, el uso generalizado de metformina y las escasas recomendaciones en relación con el déficit de vitamina B12 asociado a su uso; realizamos una revisión del tema que nos permite ubicarnos en la situación actual y tener una idea más objetiva en cuanto a las recomendaciones diagnósticas y terapéuticas.

\section{DISEÑO}

Para desarrollar esta revisión bibliográfica consultamos información disponible sobre el tema en las siguientes bases de datos científicas: PubMed, Scielo, Cochrane y Science Direct. Posterior a la recopilación de la información indexada en estas bases, seleccionamos aquellos artículos de revisión, trabajos de investigación y metaanálisis que cumplieran con el criterio de temporalidad, calidad metodológica, relevancia, validez de los resultados e impacto de las revistas en que fueron publicados.

En esta revisión se utilizaron artículos publicados hace más de 10 años con el único objetivo de exponer al lector los antecedentes del tema y cómo iniciaron las investigaciones que nos han permitido llegar hasta este punto. 
ANTECEDENTES

La asociación entre el déficit de vitamina B12 y el uso de metformina fue descrito por primera vez en 1969 por Berchtold et al. Desde entonces, se desarrollaron múltiples investigaciones sobre el tema.

En 1971, Tomkin et al, publicaron un estudio en donde se aplicaron pruebas de malabsorción intestinal a una muestra de 71 pacientes que habían utilizado metformina por 2 años o más. Posteriormente, se evaluaron sus niveles de vitamina B12 y factor intrínseco, la presencia de malabsorción de grasas, el uso de terapia antibiótica y la existencia de trastornos estructurales o de la mucosa. Concluyeron que la malabsorción de vitamina B12 era un hallazgo común en pacientes con uso prolongado de metformina, y que se asocia de forma significativa a niveles disminuidos de esta. Además, esta patología no era explicada por ninguno de los factores evaluados. Curiosamente, 28 días posteriores al retiro del medicamento, la gran mayoría de los que presentaban malabsorción se recuperaron casi en su totalidad. ${ }^{9}$

Se ha publicado gran cantidad de material sobre el tema, pero no se ha logrado un consenso en cuanto a la realización de pruebas de forma preventiva a la población que utiliza metformina de forma diaria y prolongada. Esto podría ser resultado de los pocos estudios prospectivos que se han realizado hasta la fecha.

Es de especial importancia mencionar el estudio prospectivo realizado por Aroda et al en 2016, donde se le dio seguimiento por 13 años a pacientes en tratamiento con metformina y la dosis administrada fue controlada. Tomaron en cuenta factores como: edad, sexo, índice de masa corporal (IMC), uso de inhibidores de bomba de protones o bloqueadores $\mathrm{H} 2$. Obtuvieron resultados estadísticamente significativos que relacionan el déficit de vitamina $\mathrm{B} 12$ a la dosis acumulada de metformina a través de los años. ${ }^{8}$
VITAMINA B12

La vitamina B12, también llamada cobalamina, existe en diversas formas químicas en las células donde actúa como cofactor de enzimas importantes en la síntesis de ácido desoxirribonucleico (ADN), así como en el metabolismo de aminoácidos y ácidos grasos. ${ }^{10,11}$

\section{FUENTES}

La cobalamina es sintetizada por bacterias presentes en el intestino humano. Sin embargo, la síntesis de vitamina B12 se da en sitios distales a los de absorción, por lo que el aprovechamiento es mínimo. La fuente real para el ser humano son los alimentos de origen animal como: carne, pescado y productos lácteos; mientras que las frutas, verduras y otros alimentos de origen no animal carecen de cobalamina. ${ }^{11}$

\section{ABSORCIÓN}

La absorción puede darse de dos formas: la primera ocurre por difusión pasiva a través de la superficie absortiva del tracto gastrointestinal, en la mucosa oral y nasal. Sólo 1-2\% de la dosis oral puede ser absorbida de esta forma; excepto cuando se suplementa con cantidades suprafisiológicas. ${ }^{12}$ La segunda forma es un proceso fisiológico activo, con mayor importancia e implicaciones clínicas (Ver Figura 1).

\section{FUNCIÓN METABÓLICA}

La vitamina B12 tiene dos formas activas que participan como cofactores en procesos intracelulares específicos.

\section{- Metilcobalamina}

Participa en el citoplasma como cofactor de la metionina sintasa en la formación de metionina, para la síntesis proteica, y de tetrahidrofolato, para la sintesis de ADN. ${ }^{12}$ Por esto, el déficit de 
vitamina B12 se asocia con disminución de su producción y se manifiesta como anemia megaloblástica. ${ }^{10}$

\section{- 5-desoxiadenosilcobalamina}

Actúa como cofactor de la metilmalonil coenzima A (CoA) mutasa mitocondrial que cataliza la conversión de metilmalonil CoA a succinil CoA. En situación de déficit de vitamina B12 se acumula metilmalonil CoA, que es hidrolizado a ácido metilmalónico. Este último se eleva en el plasma de los afectados y puede ser medido para corroborar el diagnóstico. ${ }^{10}$

\section{TRANSPORTE}

Las proteínas de transporte en la circulación son la haptocorrina, también llamada transcobalamina I, que se une al $70-80 \%$ de la vitamina B12 total circulante y la transcobalamina, o transcobalamina II, que transporta el $20-30 \%$ restante. ${ }^{10}$

\section{CIRCULACIÓN ENTEROHEPÁTICA}

Aproximadamente 0,5 a 5,0 microgramos por día de vitamina B12 unida a la proteína $\mathrm{R}$ son excretados en la bilis, pero más de la mitad es reabsorbida a través de los enterocitos ileales. Esto constituye un mecanismo de conservación de la vitamina que impide su pérdida diaria y retrasa la aparición de un déficit significativo ante la deprivación de esta. ${ }^{10,12}$

\section{DEFICIENCIA DE VITAMINA B12}

El cuerpo humano almacena aproximadamente 2-5 mg de vitamina B12, la mitad en el hígado. Si cesa la ingesta y/o la absorción de vitamina B12, deben transcurrir mínimo uno o dos años para desarrollar deficiencia $y$, tal vez, más hasta que se presenten manifestaciones clínicas que lo sugieran. ${ }^{14}$
Figura 1. Proceso de Absorción de la Vitamina B12

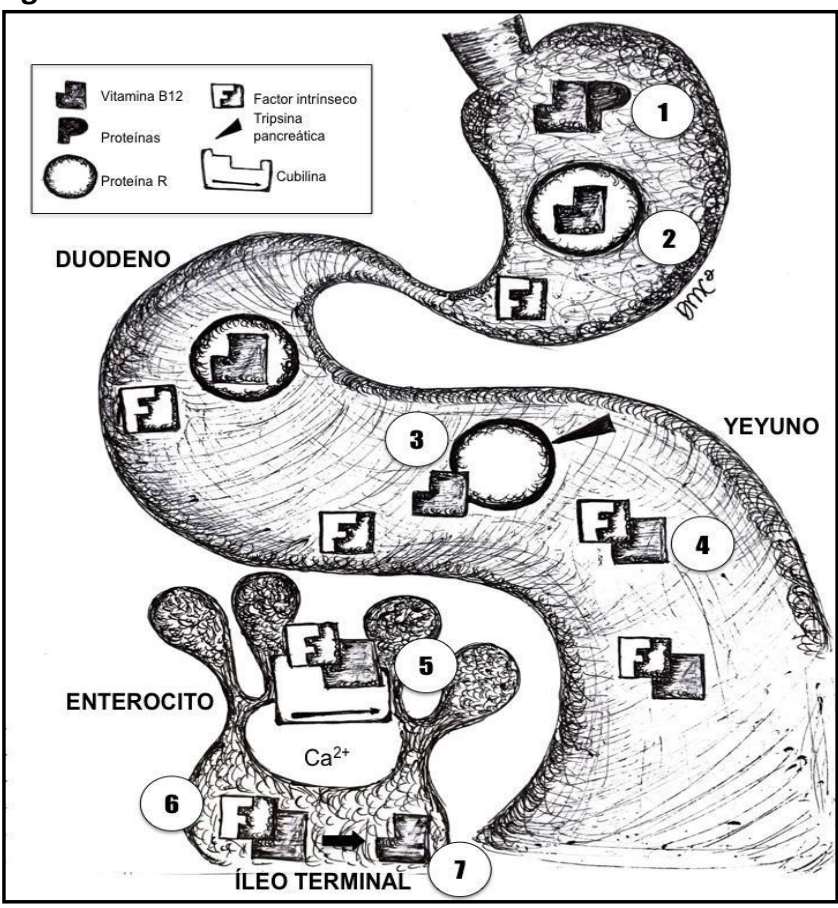

La vitamina B12 de los alimentos generalmente está unida a proteínas (1). En el estómago, por acción del ácido gástrico y la pepsina, se separan. Luego, se unen a las proteínas $R$ (2), también llamadas cobalofilinas 0 haptocorrinas, glicoproteínas presentes en el fluido gástrico y en la saliva que protegen a la vitamina del $\mathrm{pH}$ ácido del estómago ${ }^{13}$.

Una vez en el duodeno, el aumento del pH provoca la separación de la vitamina B12 y de la proteína R; esta última es degradada por la tripsina pancreática $(3)^{12}$.

Una vez liberada, la cobalamina forma un complejo con el factor intrínseco (4) (secretado por las células parietales gástricas); dicho complejo llega al íleo terminal en donde se une al receptor cubilina (5). El complejo cobalamina factor intrínseco - cubilina ingresa al enterocito por medio de endocitosis (dependiente de calcio).

La cubilina se separa y lo restante del complejo (cobalamina - factor intrínseco) llega al lisosoma (6) en donde el factor intrínseco es degradado y la cobalamina es liberada al citoplasma $(7)^{10}$. 
Si se plantea de una forma simple, la deficiencia de vitamina B12 será por disminución de la ingesta, disminución de la absorción o ambas. Un ejemplo de lo primero son las dietas vegetarianas o el veganismo estricto, el segundo caso se refiere a la interrupción, en cualquier punto, de la absorción de la vitamina; aquí se incluyen la aclorhidria, atrofia gástrica, infección persistente por Helicobacter pylori, pancreatitis, resección ileal, enfermedad de Crohn y la ingesta de medicamentos como los inhibidores de la bomba de protones y la metformina. De todos estos, es conocido que la malabsorción es la principal causa de deficiencia de vitamina B12. ${ }^{10}$

\section{PRESENTACIÓN}

Los dos sistemas más afectados son el hematológico y el neurológico. Generalmente las alteraciones neurológicas preceden a las hematológicas. El déficit debe ser severo para que se presente anemia. ${ }^{12}$

A nivel neurológico suele presentarse como parestesias simétricas en miembros inferiores, debilidad progresiva y ataxia. A nivel hematológico, se manifiesta como anemia, macrocitosis, leucopenia leve $y / o$ trombocitopenia, neutrófilos hipersegmentados en frotis de sangre periférica y ausencia de reticulocitosis. ${ }^{14}$

\section{DIAGNÓSTICO}

Ante la sospecha de deficiencia de vitamina B12, por los hallazgos tanto en la anamnesis como en el examen físico, se deben hacer estudios adicionales para confirmar o descartar el diagnóstico.

Se inicia con la biometría hemática completa con frotis de sangre periférica. Las alteraciones antes mencionadas son inespecíficas ante deficiencia de vitamina B12 y ácido fólico, por esta razón, se deben medir los niveles de cada una. ${ }^{14}$
Tabla 1: Interpretación de niveles de vitamina B12. ${ }^{14}$

\begin{tabular}{ll}
\hline Niveles $(\mathrm{pg} / \mathrm{mL})$ & Interpretación \\
\hline$>300$ & Normal \\
$200-300$ & Limítrofe \\
$<200$ & Bajo, consistente con deficiencia \\
\hline
\end{tabular}

Fuente: Clinical Manifestations and Diagnosis of Vitamin B12 and Folate Deficiency. UpToDate 2020. ${ }^{14}$

\section{METFORMINA}

La metformina es una biguanida sintética proveniente de la planta Galega officinalis, la cual ha sido utilizada desde la edad media para tratar síntomas hiperglucémicos. ${ }^{15,16}$ En 1922 se descubre su principal componente: la dimetil biguanida. No obstante, es opacado por la síntesis contemporánea de insulina. ${ }^{17}$ No fue hasta la década de 1950 en que Jean Sterne estudia sus propiedades y subsecuentemente desarrolla el glucófago. ${ }^{16,17}$ A partir de esta época diversos países inician la aprobación del medicamento como tratamiento para hiperglicemia. ${ }^{15,16}$

Actualmente la metformina es uno de los tratamientos más utilizados para DM 2. También está indicada en pacientes con síndrome de ovario poliquístico y síndrome metabólico. ${ }^{15} \mathrm{Ha}$ demostrado mejorar el índice glicémico, producir pérdida de peso o neutralidad de este; desencadenar pocos episodios de hipoglicemia y reducir el riesgo cardiovascular. Todo esto a un bajo costo y un perfil farmacológico seguro. ${ }^{16}$

Su mecanismo de acción no está completamente dilucidado a pesar de la larga experiencia en su uso y las numerosas investigaciones sobre ella. El mecanismo más aceptado es a nivel hepático, en donde inhibe la gluconeogénesis al bloquear procesos mitocondriales. ${ }^{16,18}$ También aumenta la utilización de 
glucosa por el músculo esquelético, aumenta la sensibilidad a la insulina, inhibe la absorción intestinal de glucosa y genera otros efectos beneficiosos. ${ }^{15}$ Esto se atribuye a su efecto pleiotrópico. ${ }^{18}$

La metformina se administra por vía oral. Se absorbe de manera lenta en el lumen del intestino delgado debido a su naturaleza hidrofílica, por lo cual la difusión pasiva a través de la membrana es mínima. ${ }^{15,16}$ Su concentración en el yeyuno es de 500 $\mathrm{mcg} / \mathrm{g}, 300$ veces mayor a la del plasma. La biodisponibilidad varía entre 50 a $60 \%$, y es afectada por aumento de la motilidad gástrica y alimentos con alto contenido graso. ${ }^{15,17}$

Interactúa con múltiples receptores intestinales para su absorción. Estos son: el transportador de cationes orgánicos tipo 1 (OCT-1) y transportador de cationes orgánicos tipo 3 (OCT-3) en un 25\%, el transportador de monoaminas de la membrana plasmática (PMAT) en un $20 \%$, el transportador de serotonina (SERT) en otro $20 \%$ y el porcentaje restante por numerosos receptores. El OCT-1 probablemente determina la concentración luminal de la metformina. ${ }^{16}$

La absorción hepática se da activamente a través de OCT-1. La metformina no es metabolizada, no genera metabolitos ni conjugados. ${ }^{15}$ Un 30 a $50 \%$ de la dosis es eliminada sin cambios a través de la orina, mientras que un $30 \%$, también sin cambios, a través de las heces. La excreción requiere transportadores renales como el transportador de cationes orgánicos tipo 2 (OCT-2). ${ }^{15,16}$

Hay evidencia que la metformina altera el microbioma intestinal disminuyendo la diversidad bacteriana de la misma. Además, hay aumento del número de células productoras de mucina. Se piensa que estos cambios pueden favorecer los efectos adversos gastrointestinales y alteraciones en la farmacodinamia de otros medicamentos. No obstante, no se ha logrado establecer una relación significativa. ${ }^{15}$

Los efectos adversos más comunes son los gastrointestinales, como diarrea y náuseas. Los mismos se pueden relacionar a la exposición intestinal excesiva a serotonina. Se propone el transporte reducido de este al interior de la célula, ya que la metformina interactúa con los receptores SERT y OCT1. La acidosis láctica, otro efecto adverso descrito, es secundaria al aumento de captación y utilización de glucosa. El tema de esta revisión, la deficiencia de vitamina $B 12,{ }^{15,16}$ se analizará a continuación.

\section{DÉFICIT DE VITAMINA B12 ASOCIADO A METFORMINA}

Conocer el mecanismo de absorción de la vitamina B12 en el cuerpo humano permite identificar la parte del proceso en donde la metformina interfiere con la absorción de la vitamina B12.

Teorías previamente propuestas consistían en que el consumo de metformina producía sobrecrecimiento bacteriano favoreciendo así la unión del complejo cobalamina - factor intrínseco a bacterias en lugar de ser absorbida. Otra teoría afirmaba que la metformina alteraba la motilidad intestinal por lo que se reducía la absorción de la vitamina. ${ }^{10}$ También se propuso la reducción de niveles de factor intrínseco. ${ }^{19}$

La teoría más aceptada es que la metformina actúa como antagonista de los cationes de calcio necesarios para la interacción entre el complejo cobalamina factor intrínseco y la cubilina, que en condiciones normales daría paso a la entrada de la vitamina al enterocito. ${ }^{10}$

La literatura describe numerosos factores que pueden predisponer a un individuo a desarrollar este déficit vitamínico, incluyendo el uso de metformina, dosis y 
la duración del tratamiento. La gran mayoría de los estudios toman en cuenta factores propios del paciente, como la edad, sexo e IMC. También incluyen el uso de ciertos medicamentos o sustancias concomitantes a la metformina. A continuación, se describen los principales factores estudiados y cómo impactan en el desarrollo de esta enfermedad.

\section{$E D A D$}

Las personas mayores de 75 años tienen riesgo de tener niveles bajos de vitamina B12 por ingesta inadecuada, malabsorción y mayor incidencia de anemia perniciosa ${ }^{20,21}$. Esto se ha descrito en diversos estudios, donde los pacientes de mayor edad son más propensos a tener deficiencia de esta vitamina independientemente del uso de metformina. Sin embargo, su uso aumenta las probabilidades de deficiencia $22,23,24$.

\section{SEXO}

Esta variable es descrita en todos los estudios revisados. En algunos la población femenina es mayor $8,22,23,25,26$. En otros, la masculina; como por ejemplo el estudio de los veteranos en donde representaban el $95 \%$ de la población estudiada ${ }^{27,28,29,30}$. Ningún estudio ha demostrado que esta variable represente un factor de riesgo o protector para el desarrollo de deficiencia de vitamina B12 $8,22,23,25,26,27,28,29,39$.

\section{ÍNDICE DE MASA CORPORAL (IMC)}

El estudio prospectivo con más años de seguimiento no describe relación entre niveles bajos de vitamina B12 e IMC en pacientes tratados con metformina. ${ }^{8}$ De forma similar, un estudio realizado en Sudáfrica en 2016 con 121 participantes y otro en Corea del Sur con 1111 pacientes no demostraron diferencia estadísticamente significativa en esta relación. ${ }^{26,28}$
Un hallazgo interesante se describe en el estudio realizado por Nervo et al en 2010 , donde se establece una relación directamente proporcional entre el peso y niveles de cobalamina en pacientes tratados con metformina. ${ }^{31}$

\section{INHIBIDORES DE LA BOMBA DE PROTONES $Y$ ANTAGONISTAS DE RECEPTOR H2.}

Se ha planteado una relación entre inhibidores de la bomba de protones (IBP), antagonistas del receptor $\mathrm{H} 2$ y déficit de vitamina B12. Este grupo de medicamentos suprime la secreción de ácido gástrico, afectando el proceso de absorción de la vitamina B12. ${ }^{10}$

Su uso es un criterio de exclusión en la mayoría de los casos, ya que puede ser un factor confusor al evaluar los efectos de la metformina por si sola. Otros estudios lo toman en cuenta para medir su capacidad de afectar la absorción de vitamina B12.

Damiao et al describen que los pacientes diabéticos que utilizaban metformina de forma concomitante con IBP o antagonistas $\mathrm{H} 2$ presentaron niveles más bajos de cobalamina en comparación con aquellos que solamente utilizaban metformina, siendo este hallazgo estadísticamente significativo. ${ }^{32}$

Por otro lado, los estudios de Nervo et al y Aroda et al no encontraron asociación estadísticamente significativa entre el uso de IBP o antagonistas $\mathrm{H} 2$ y niveles de vitamina B12. ${ }^{8,31}$

\section{INGESTA DE ALCOHOL}

La ingesta excesiva de alcohol está descrita como una causa de déficit de vitamina B12, siendo muchas veces un criterio de exclusión. Para categorizar el consumo como excesivo, utilizan diversas herramientas como el cuestionario AUDIT, por sus siglas en inglés (Alcohol Use Disorders Identification Test) o individuos con 
consumo mayor de 1 a 2 bebidas por día, entre otras. ${ }^{19,20,23}$

En los estudios en los que incluyeron individuos con esta característica, ocurrían tres cosas: no había diferencia significativa entre los grupos de estudio con o sin deficiencia de la vitamina B12, de haber diferencia entre se catalogaba como factor de confusión para un mejor análisis de las variables del estudio o no se tomaba en cuenta; por lo que puede jugar el papel de factor de confusión, alterando la certeza de los resultados. $22,23,28,29,30$

\section{DOSIS DE METFORMINA}

La dosis diaria de metformina constituye uno de los factores más estudiados en relación con el déficit de Vitamina B12. Diversas investigaciones han demostrado una asociación entre el consumo de metformina y disminución en las concentraciones séricas de esta vitamina, pero que no necesariamente se asocian a un déficit de la misma. ${ }^{34,35}$

Se ha descrito que a mayor dosis de metformina, mayor es la diminución de vitamina B12 y, por ende, aumenta la probabilidad de presentar un déficit. Por cada aumento de $1 \mathrm{mg}$ en la dosis diaria de metformina, hay una reducción en los niveles de vitamina B12 de $0.042 \mathrm{pg} / \mathrm{mL} .{ }^{36}$

No existe un valor de corte establecido para hablar de riesgos. Sin embargo, los estudios sugieren que a partir de dosis mayor o igual 1500 mg/día de metformina, incrementa significativamente la probabilidad de disminuir los niveles séricos de cobalamina como lo demostraron Kim et al. ${ }^{28}$

\section{DURACIÓN DE TRATAMIENTO}

Existen múltiples reportes que indican que la disminución de vitamina B12 asociada al uso de metformina puede presentarse con apenas 3 a 4 meses de tratamiento. Sin embargo, la mayoría de los estudios han reportado que el déficit significativo solo ocurre luego de al menos 5 a 10 años de tratamiento. ${ }^{37}$

Un metaanálisis, realizado en China en el 2014, encontró niveles bajos de cobalamina en pacientes que tenían menos de 3 años de tratamiento, dejando en evidencia que las alteraciones pueden verse desde etapas tempranas. ${ }^{38}$ Otro metaanálisis que incluyó 31 estudios, demostró nuevamente la relación inversamente proporcional entre los meses de tratamiento y los niveles séricos de vitamina B12. Fue evidente desde el primer año de tratamiento, pero solo aquellos con más de 3 años de uso tenían riesgo de presentar un déficit clínico. ${ }^{39}$

\section{DURACIÓN DE LA ENFERMEDAD}

La duración de la DM 2 en los pacientes con déficit de vitamina B12 ha sido un aspecto ampliamente estudiado; no obstante, no se ha demostrado una verdadera relación entre ambos. En un estudio realizado en Corea del Sur donde la población estudiada presentaba una duración media de la enfermedad de $11.3 \pm 7.9$ años; no se encontró relación con los niveles de vitamina B12. Sin embargo, se demostró una relación estadísticamente significativa entre el desarrollo de anemia y los años de la enfermedad. ${ }^{22}$

En otro estudio realizado en Sudáfrica encontraron que la mayoría de los pacientes con deficiencia de vitamina B12 eran aquellos con mayor tiempo de enfermedad. Pese a esto, la diferencia con el grupo control no fue estadísticamente significativa. ${ }^{26}$

Existe evidencia que vincula la dosis acumulada de metformina y la presencia de neuropatía periférica a un mayor riesgo de presentar déficit de cobalamina. Ambas situaciones pueden estar presentes en fases avanzadas de la enfermedad y, por esto, no se puede descartar que la duración de la enfermedad sea un 
factor de riesgo para su presencia y debe tomarse en cuenta a la hora de la evaluación de un paciente. ${ }^{8,25}$

\section{MULTIVITAMINAS}

Son el suplemento más utilizado en los países desarrollados, populares en la población adulta y utilizados con el objetivo de mantener un adecuado estado de salud. ${ }^{40}$ En los estudios para determinar la deficiencia de vitamina B12 secundaria a la utilización de metformina muchas veces son consideradas un factor de exclusión. ${ }^{25,26}$ Kancherla et al, en el estudio de veteranos, no las toman como variable, pero reconocen que su uso pudo haber impactado en los resultados. ${ }^{27}$

De los estudios que la incluyeron como variable, Ko et al demostraron que su uso no interviene en el desarrollo de deficiencia de vitamina B12. ${ }^{22}$ Sin embargo, estudios han comprobado que las mismas funcionan como un factor protector en pacientes diabéticos en tratamiento con metformina. ${ }^{28,30} \mathrm{Kim}$ et al demuestran que su uso se asocia a una menor prevalencia (OR: 0.14) y recomiendan su uso concurrente. $^{28}$

\section{SITUACIÓN ACTUAL}

Desde que se publicó el primer artículo que vinculó el uso de metformina con déficit de vitamina B12 y a pesar de la evidencia existente, aun no se ha llegado a un consenso en cuanto a la realización de pruebas de tamizaje en la población en riesgo.

Recientemente fue publicado un estudio realizado en la India en el cual se aplicó a una población expuesta a metformina un instrumento llamado Índice de Uso de Metformina (MUI, por sus siglas en inglés). Se demostró que aquellos con un $\mathrm{MUI}>5$ tenían mayor riesgo de presentar déficit de vitamina B12 en comparación a los que presentaron $\mathrm{MUI}<5$. Por esta razón, se propuso que al aplicarse esta herramienta se tome como valor de corte un $\mathrm{MUI}>5$ para iniciar suplementación, pues en el contexto de su área de estudio, conllevaba un gran costo el aplicar pruebas serológicas. ${ }^{19}$

$M U I=\frac{\text { Dosis de metformina } \quad(m g) x \text { Duración de tratamiento (años) }}{1000}$

\section{CONCLUSIONES}

La metformina representa uno de los principales tratamientos para DM 2 a nivel mundial. Sus efectos adversos son bien conocidos, principalmente los de tipo gastrointestinal. El déficit de vitamina B12 ha sido descrito como un efecto adverso que puede impactar en la calidad de vida de los pacientes.

El mecanismo mediante el cual la metformina disminuye los niveles de cobalamina aún no se encuentra muy bien dilucidado. Se ha propuesto la alteración del microbioma intestinal o el aumento de la motilidad como causas. La teoría más aceptada al momento es que antagoniza al calcio en el proceso de absorción de la vitamina B12 por el enterocito.

Factores como la dosis y la duración del tratamiento con metformina son los que se asocian de manera significativa a la disminución y/o deficiencia de vitamina B12. En la actualidad no existen guías que establezcan indicaciones para tamizaje de este déficit en usuarios de metformina. Sin embargo, múltiples estudios han demostrado y sugieren la monitorización de los niveles de cobalamina, sobre todo en pacientes con altas dosis y larga duración de consumo de metformina. 


\section{PUNTOS CLAVES}

- Tanto la absorción de la cobalamina como el mecanismo de acción de la metformina son procesos complejos que aún están siendo estudiados.

- La teoría más aceptada para el déficit de vitamina B12 por el consumo de metformina es el antagonismo de calcio, necesario en la interacción entre el complejo cobalamina factor intrínseco y la cubilina, y su consecuente absorción por el enterocito ileal.

- El tratamiento con metformina por más de 3 años establece la posibilidad de un déficit vitamínico.

- A mayor dosis de metformina mayor riesgo de desarrollar disminución de vitamina B12 y,

\section{BIBLIOGRAFÍA}

1. Kanter J, Bornfeldt K. Impact of Diabetes Mellitus | Arteriosclerosis, Thrombosis, and Vascular Biology [Internet]. 2016 [Consultado 4 Jun 2020] 36:1049-1053 Disponible en: https://www.ahajournals.org/doi/10.1161/ATVBAHA.116.30730 2.

2. Guthrie RA, Guthrie DW. Pathophysiology of diabetes mellitus. Crit Care Nurs Q [Internet]. 2004 [Consultado 28 May 2020];27(2):113-125. Disponible https://pubmed.ncbi.nlm.nih.gov/15137354/

3. International Diabetes Federation. IDF Diabetes Atlas, 9th edn. Brussels, Belgium [Internet]: 2019 [Consultado 28 May 2020]. Disponible en: https://www.diabetesatlas.org

4. American Diabetes Association. Standards Of Medical Care in Diabetes. The Journal of CliniCal and applied research and education [Internet]. 2020 [Consultado 28 May 2020]. Disponible en:

https://care.diabetesjournals.org/content/diacare/suppl/2019/ 12/20/43.Supplement_1.DC1/Standards_of_Care_2020.pdf

5. Sanchez-Rangel E, Inzucchi SE. Metformin: clinical use in type 2 diabetes. Diabetologia [Internet]. 2017 [Consultado 30 May consecuentemente, deficiencia. Principalmente en dosis mayores a $1500 \mathrm{mg} /$ día.

- Las multivitaminas han demostrado prevenir la disminución y el déficit de cobalamina en múltiples estudios. Son consideradas un factor protector.

- El tamizaje serológico es recomendado en pacientes que presenten un mayor riesgo de desarrollar esta deficiencia.

- Se ha propuesto el Índice de Uso de Metformina (MUI) como herramienta de tamizaje sin la necesidad de realizar pruebas serológicas. Constituye un recurso costo efectivo y reproducible en otras poblaciones.

2020];60(9):1586-1593. Disponible

https://pubmed.ncbi.nlm.nih.gov/28770321/

6.Viollet B, Guigas B, Sanz Garcia N, Leclerc J, Foretz M, Andreelli F. Cellular and molecular mechanisms of metformin: an overview. Clin Sci (Lond) [Internet]. 2012 [Consultado 30 May 2020];122(6):253-270. doi:10.1042/CS20110386. Disponible en: https://pubmed.ncbi.nlm.nih.gov/22117616/

7. Shipton MJ, Thachil J. Vitamin B12 deficiency - A 21st century perspective. Clin Med (Lond) [Internet]. 2015 [Consultado 30 May 2020];15(2):145-150. Disponible en: https://pubmed.ncbi.nlm.nih.gov/25824066/

8. Aroda VR, Edelstein SL, Goldberg RB, et al. Long-term Metformin Use and Vitamin B12 Deficiency in the Diabetes Prevention Program Outcomes Study. J Clin Endocrinol Metab [Internet]. 2016 [Consultado 30 May 2020];101(4):1754-1761. Disponible en: https://pubmed.ncbi.nlm.nih.gov/26900641/

9. Tomkin GH, Hadden DR, Weaver JA, Montgomery DA. VitaminB12 status of patients on long-term metformin therapy. Br Med J [Internet]. 1971 [Consultado 30 May 2020];2(5763):685-687. Disponible en: https://www.ncbi.nlm.nih.gov/pmc/articles/PMC1796258/ 
10. Ahmed MA. Metformin and Vitamin B12 Deficiency: Where Do We Stand? J Pharm Pharm Sci [Internet]. 2016 [Consultado 30 May 2020];19(3):382-398. Disponible en: https://pubmed.ncbi.nlm.nih.gov/27806244/

11. A. Victor Hoffbrand. Anemias Megaloblásticas. Jameson J, Fauci A, Kasper D. Harrison Principios de Medicina Interna Capítulo 95. 20th ed. Ciudad de México: McGraw-Hill; 2018. p699

12. Allen LH, Miller JW, de Groot L, et al. Biomarkers of Nutrition for Development (BOND): Vitamin B-12 Review. J Nutr [Internet]. 2018 [Consultado 30 May 2020];148(suppl_4):1995S-2027S. doi:10.1093/jn/nxy201

Disponible en: https://pubmed.ncbi.nlm.nih.gov/30500928/

13. Forrellat Barrios Mariela, Gómis Hernández Irma, Gautier du Défaix Gómez Hortensia. Vitamina B12: metabolismo y aspectos clínicos de su deficiencia. Rev Cubana Hematol Inmunol Hemoter [Internet]. 1999 Dic [citado 2020 Jun 03] ; 15(3): 159174. Disponible en: http://scielo.sld.cu/scielo.php?script=sci arttext\&pid=S0864$\underline{02891999000300001 \& \operatorname{lng}=\mathrm{es}}$.

14. Clinical Manifestations and Diagnosis of Vitamin B12 and Folate Deficiency. Means R, Fairfield K. UpToDate [Internet]. Uptodate.com. 2020 [citado 2020 Jun 03]. Disponible en: https://www.uptodate.com/contents/clinical-manifestationsand-diagnosis-of-vitamin-b12-and-folatedeficiency?csi=0e3d01bd-d8ec-4735-914a-

85aebd0d494b\&source=contentShare

15. Markowicz-Piasecka M, Huttunen KM, Mateusiak L, MikiciukOlasik E, Sikora J. Is Metformin a Perfect Drug? Updates in Pharmacokinetics and Pharmacodynamics. Curr Pharm Des [Internet]. 2017 [Citado 29 may 2020];23(17):2532-2550. Disponible: http://www.eurekaselect.com/147864/article

16. McCreight LJ, Bailey CJ, Pearson ER. Metformin and the gastrointestinal tract. Diabetologia [Internet]. 2016 [Citado 29 may 2020];59(3):426-435.

Disponible: https://www.ncbi.nlm.nih.gov/pmc/articles/PMC4742508/

17. Thomas I, Gregg B. Metformin; a review of its history and future: from lilac to longevity. Pediatr Diabetes [Internet]. 2017 [Citado 29 may 2020];18(1):10-16. Disponible: https://deepblue.lib.umich.edu/bitstream/handle/2027.42/135 516/pedi12473.pdf?sequence=2\&isAllowed=y

18. Flory J, Lipska K. Metformin in 2019. JAMA [Internet]. 2019 [Citado 29 may 2020];321(19):1926-1927. Disponible: https://jamanetwork.com/journals/jama/articleabstract/2731896

19. Shivaprasad C, Gautham K, Ramdas B, Gopaldatta KS, Nishchitha K. Metformin Usage Index and assessment of vitamin B12 deficiency among metformin and non-metformin users with type 2 diabetes mellitus [published online ahead of print, 2020 Apr 7]. Acta Diabetol [Internet]. 2020;10.1007/s00592-02001526-4. doi:10.1007/s00592-020-01526-4. Disponible en: https://pubmed.ncbi.nlm.nih.gov/32266492/

20. Langan RC, Goodbred AJ. Vitamin B12 Deficiency: Recognition and Management. Am Fam Physician [Internet]. 2017 [Citado 29 may 2020];96(6):384-389. Disponible en: https://pubmed.ncbi.nlm.nih.gov/28925645/?dopt=Abstract

21. Sukumar N, Saravanan P. Investigating vitamin B12 deficiency. BMJ [Internet]. 2019 [Consultado 28 May 2020];:I1865. Disponible en: https://www.bmj.com/content/365/bmj.I1865

22. Ko SH, Ko SH, Ahn YB, et al. Association of vitamin B12 deficiency and metformin use in patients with type 2 diabetes. J Korean Med Sci [Internet]. 2014 [Consultado 30 May 2020];29(7):965-972. Disponible en: https://pubmed.ncbi.nlm.nih.gov/25045229/

23. Kang D, Yun JS, Ko SH, et al. Higher prevalence of metformininduced vitamin B12 deficiency in sulfonylurea combination compared with insulin combination in patients with type 2 diabetes: a cross-sectional study. PLoS One [Internet]. 2014 [Consultado 28 May 2020];9(10):e109878. Published 2014 Oct 9. Disponible en: https://pubmed.ncbi.nlm.nih.gov/25299054/

24. Owhin SO, Adaja TM, Fasipe OJ, Akhideno PE, Kalejaiye OO et al. Prevalence of vitamin $B_{12}$ deficiency among metformintreated type 2 diabetic patients in a tertiary institution, SouthSouth Nigeria. SAGE Open Med [Internet]. 2019 [Consultado 28 May 2020]; 7:2050312119853433. Published 2019 May 23. Disponible en: https://www.ncbi.nlm.nih.gov/pmc/articles/PMC6535731/

25. Alvarez M, Sierra OR, Saavedra G, Moreno S. Vitamin B12 deficiency and diabetic neuropathy in patients taking metformin: a cross-sectional study. Endocr Connect [Internet]. 2019 [Citado 29 may 2020];8(10):1324-1329. Disponible: https://www.ncbi.nlm.nih.gov/pmc/articles/PMC6790897/

26. Ahmed MA, Muntingh G, Rheeder P. Vitamin B12 deficiency in metformin-treated type-2 diabetes patients, prevalence and association with peripheral neuropathy. BMC Pharmacol Toxicol 
[Internet]. 2016 [Citado 29 may 2020];17(1):44. Disponible: https://www.ncbi.nlm.nih.gov/pmc/articles/PMC5054613/

27. Kancherla V, Elliott JL Jr, Patel BB, et al. Long-term Metformin Therapy and Monitoring for Vitamin B12 Deficiency Among Older Veterans. J Am Geriatr Soc [Internet]. 2017 [Citado 29 may 2020];65(5):1061-1066.

https://www.ncbi.nlm.nih.gov/pmc/articles/PMC5435502/

28. Kim J, Ahn CW, Fang S, Lee HS, Park JS. Association between metformin dose and vitamin B12 deficiency in patients with type 2 diabetes. Medicine (Baltimore) [Internet]. 2019 [Citado 29 may 2020];98(46):e17918.

https://www.ncbi.nlm.nih.gov/pmc/articles/PMC6867725/

29. Iftikhar R, Kamran SM, Qadir A, Iqbal Z, bin Usman H. Prevalence of vitamin B12 deficiency in patients of type 2 diabetes mellitus on metformin: a case control study from Pakistan. Pan Afr Med J [Internet]. 2013 [Citado 29 may 2020];16:67. Disponible: https://www.ncbi.nlm.nih.gov/pmc/articles/PMC3976659/\#: :t ext=There\%20was\%20a\%20significant\%20correlation, patients) \%20(Figure\%201).

30. Khan A, Shafiq I, Hassan Shah M. Prevalence of Vitamin B12 Deficiency in Patients with Type II Diabetes Mellitus on Metformin: A Study from Khyber Pakhtunkhwa. Cureus [Internet]. 2017 [Citado 29 may 2020];9(8):e1577. Disponible: https://www.ncbi.nlm.nih.gov/pmc/articles/PMC5647126/

31. Nervo M, Lubini A, Raimundo FV, et al. Vitamin B12 in metformin-treated diabetic patients: a cross-sectional study in Brazil. Rev Assoc Med Bras (1992) [Internet]. 2011 [Consultado 29 May2020];57(1):46-49. Disponible en: https://pubmed.ncbi.nlm.nih.gov/21390459/

32. Damião CP, Rodrigues AO, Pinheiro MF, et al. Prevalence of vitamin B12 deficiency in type 2 diabetic patients using metformin: a cross-sectional study. Sao Paulo Med J [Internet]. 2016 [Consultado 29 May 2020];134(6):473-479. Disponible en: https://www.scielo.br/scielo.php?script=sci_arttext\&pid=S1516 $-31802016005004103 \& \operatorname{lng}=e n \& n r m=i s o \& \ln g=e n$
33. Biemans $\mathrm{E}$, Hart HE, Rutten GE, Cuellar Renteria VG, Kooijman-Buiting AM, Beulens JW. Cobalamin status and its relation with depression, cognition and neuropathy in patients with type 2 diabetes mellitus using metformin. Acta Diabetol [Internet]. 2015 [Consultado 29 May 2020];52(2):383-393. Disponible en: https://pubmed.ncbi.n/m.nih.gov/25315630/

34.Niafar M, Hai F, Porhomayon J, Nader ND. The role of metformin on vitamin B12 deficiency: a meta-analysis review. Intern Emerg Med [Internet]. 2015 [Consultado 2 Jun 2020];10(1):93-102. Disponible en: https://pubmed.ncbi.nlm.nih.gov/25502588/

35. Chapman LE, Darling AL, Brown JE. Association between metformin and vitamin B12 deficiency in patients with type 2 diabetes: A systematic review and meta-analysis. Diabetes Metab [Internet]. 2016 [Consultado 2 Jun 2020];;42(5):316-327. Disponible en: https://pubmed.ncbi.nlm.nih.gov/27130885/

36. Beulens JW, Hart HE, Kuijs R, Kooijman-Buiting AM, Rutten GE. Influence of duration and dose of metformin on cobalamin deficiency in type 2 diabetes patients using metformin. Acta Diabetol [Internet]. 2015 [Consultado 3 Jun 2020];52(1):47-53. Disponible en: https://pubmed.ncbi.nlm.nih.gov/24908579/

37. Wile DJ, Toth C. Association of metformin, elevated homocysteine, and methylmalonic acid levels and clinically worsened diabetic peripheral neuropathy. Diabetes Care [Internet]. 2010 [Consultado 3 Jun 2020];33(1):156-161. Disponible en: https://pubmed.ncbi.nlm.nih.gov/19846797/

38.Liu Q, Li S, Quan H, Li J. Vitamin B12 status in metformin treated patients: systematic review. PLoS One [Internet]. 2014 [Consultado 2 Jun 2020];9(6):e100379. Published 2014 Jun 24. Disponible en: https://pubmed.ncbi.nlm.nih.gov/24959880/

39. Yang W, Cai X, Wu H, Ji L. Associations between metformin use and vitamin $B 12$ levels, anemia, and neuropathy in patients with diabetes: a meta-analysis. J Diabetes [Internet]. 2019 [Consultado 3 Jun 2020];11(9):729-743. Disponible en: https://pubmed.ncbi.nIm.nih.gov/30615306/ 